\section{SOI: $1.1 /$ TAS DOI: $10.15863 / \mathrm{TAS}$ \\ International Scientific Journal Theoretical \& Applied Science}

p-ISSN: 2308-4944 (print) e-ISSN: 2409-0085 (online)

Year: $2015 \quad$ Issue: $05 \quad$ Volume: 25

Published: $30.05 .2015 \quad$ http://T-Science.org
EInur Latif oglu Hasanov

Corresponding member of International Academy of Theoretical and Applied Sciences,

Ph.D. postgraduate Institute of Local-lore of Ganja Branch Azerbaijan National Academy of Sciences, Ganja, Azerbaijan 1-hasan@hotmail.com

SECTION 12. Geology. Anthropology.

Archaeology.

\title{
ABOUT SCIENTIFIC RESEARCH OF HERITAGE OF MIRZA SHAFI VAZEH
}

Abstract: In this scientific article were researched the basic innovative arguments about heritage of Mirza Shafi Vazeh on the basis of literary and historical facts.

Key words: Mirza Shafi Vazeh, Ganja, innovative arguments, Azerbaijan, poet.

Language: French

Citation: Hasanov EL (2015) ABOUT SCIENTIFIC RESEARCH OF HERITAGE OF MIRZA SHAFI VAZEH. ISJ Theoretical \& Applied Science 05 (25): 34-37.

Soi: http://s-o-i.org/1.1/TAS*05(25)7 Doi: crossef http://dx.doi.org/10.15863/TAS.2015.05.25.7

\section{L'ETUDE SCIENTIFIQUE DU PATRIMOINE DU MIRZÉ CHAFI VAZEH}

Résumé: Dans cet article scientifique ont étudié les arguments de base innovantes sur le patrimoine de Mirzé Chafi Vazeh sur la base de faits littéraires et historiques.

Mots-clés: Mirzé Chafi Vazeh, Gandja, arguments novateurs, Azerbaïdjan, poète.

\section{La introduction}

L'ordre du président de la république datée de 3 février 2014 consernant la célébration de 220 e anniversaire de Mirzé Chafi Vazeh dresse une grande obligation devant les chercheurs de Mirza Shafi.

Mirzé Chafi Vazeh est né en 1794 à Gandja dans une famille d'un maçon qui s'appelait Karbalai Sadiq. Il s'occupait de travaux architecturaux dans le palais du Khan de Gandja Djavad Khan. C'est pourqoui les vers de Mirzé Chafi Vazeh était respectés en étranger dans cette époque - là et ceux ci fait connaître leur auteur. A propos ses oeuvres ne sont pas gardés en Azerbaïdjan, il n'a pas été connu dans son pays natal. $M$. Chafi a perdu son père quand il était très jeune. Il a vécu sous les auspices de son parent Hadji Abdulla. Son fils devient un clergé. Chafi a commencé à faire ses études réligieuses. Mais comme il s'intéressait à l'enseignement laïque, dans le medrécé (une école réligieuse) les clergés ont refusé de lui enseigner.

L'un des premiers éducateurs du XIX siècle Mirzé Chafi Vazeh était contemporain de Abbasqoulou Aga Bakikhanov et dévancier de Mirze Fatali Akhundov. La poésie lyrique et philosophique avait été connue en Europe et en Russie. Ses vers rappelaient aux lecteurs europeens et aux lecteurs russes le grand poète persan Eumar Khaïam. C'est pourqoui les vers de Mirzé Chafi Vazeh était respectés en étranger dans cette époque - là et ceux ci fait connaître leur auteur. A propos ses oeuvres ne sont pas gardés en Azerbaïdjan, il n'a pas été connu dans son pays natal.

En fait Mirze Chafi Vazeh a dû quitter le medrécé. Sur cet événement orientimaliste Adolf Berjé a écrit dans ses mémoires. Après avoir quitté le medrécé il a commencé à s'instruire à developper ses connaissances intellectuelles et il a apris la langue persanne. Ça lui a permis de connaitre les grands poètes de l'Orient et ses oeuvres. A l'aide de Hadji Abdoulla Mirze Chafi Vazeh dirigeait les travails du palais de Pusté Khanoum, la fille de Djavad Khan.

En 1826 pendant la guerre entre la Russie et 1'Iran Pusté Khanoum est oubligée de s'en fuire à l'Iran avec son frère Ougourlou Khan. Mirza Chafi reste sans travail, en ce moment-là Hadji Abdoulla meurt. Malgré que Mirzé Chafi reste seul, il ne tombe pas dans la déprétion, il commence à enseigner aux enfants et s'occupe d'apprendre l'écriture aux enfants. Un de ces enfants était Mirzé Fétéli Akhoundov qui apprenait l'écriture.

En 1840 Mirza Chafi Vazeh arrive de Gandja à Tiflis. Au mois de novembre de la même année à l'aide de Mirza Fatali Akhundov il est nommé le 
professeur d' azerbaijanais et persan à l'école de territoire à Tiflis. Il habite à Tiflis jusqu 'a la fin de l'année 1846. Après la sépartion de Tiflis il a écrit la poésie "Adieu Tiflis" en langue persanne. En 1844 il crée une colloque pilosophique, littéraire Divanihikmet à Tiflis. A ce temps là, dans le colloque de vers participaient Abbaskoulau aga Bakikhanov. M.F.Akhoundov, les poètes Najami, Cheula, Mirza Hassan, Hadji Abdulla, Mirza Yousif, Vidadi, Haci Yusif. Grâce à l'activité de cette colloque Mirza Chafi Vazeh à fait la connaissace avec les hommes de science célèbres et hommes de profession (mitier).

\section{Gandja et Mirzé Chafi Vazeh}

L'un des membres de cette colloque était Fridrikh Fon Bodenchtedt né en 1819, au Province Payné se trouvant à côté de Honnover, la captale de Bas Sakson de'Allemagne. Bodenchtedtétait juif. Il avait fait ses études dans les universités célèbres comme Guétinguène, Munhène, Berlin. En 1841, Bodenchtetd arrive à Moscou et la il s'occupe de l'éducation des enfants de knyaz Mikhail Qolitsin. En 1844, avec l'inivitation de générale Neydtqart, juge en tête de caucase, il arrive à Tiflis et y travaille en qualité de professeur. Il apprenait la langue persanne. Mirza Chafi a son tour, faisait la connaissance avec les oeurvres des classiques de 1 'europe d'ouest, a l'aide de lui. En 1846 Bodenchtedt rentre en Allemagne. Dans les années 1889- 1890 il va aux Etats - Unis. En 1892 il est mort et il est enterré dans la ville Visbaden de l'Allemagne. En 1846, Mirza Chafi rentre à Gandja. Il est nommé en comme le professeur à l'école "Qəza" (territoire) ouverte par l'état Attenant à professeurat, il s'occupe aussi de la créativité de poésie. En 1850, au mois de janvier il rentre à Tiflis et commence à travailler en qualité de professeur de la langue azerbaidjanaise à la gymnase des nobles. Il y habite jusqu à la mort (à la fin de sa vie). En 1852, le 28 novembre, il est derrière du jardin Botanique. Aujourd'hui, il n'y a pas d'originaire en persan et azerbaïdjanais édité en russe et dans les langues de l'europe d'ouest. Les manuscrits des vers sont emportés en Allemangne par Fridrix fon Boudenchtedt.

Une partie de ces poésies ont étéintroduites dans son oeuvre "Mille et un jour à l'Est". Après son oeuvre, en 1851, F. Bodenstedt fait publier à Berlin un petit livret nommé Les chants de Mirzé Chéfi. Celui-ci, après une diffusion rapide, rend son auteur très célèbre. Peu après les oeuvres de Mirzé Chéfi se répandent vite dans toute l'Europe Occidentale.Outre l'allemand, elles sont traduites en anglais, français, italien, suisse, norvégien,holland,danois, polognais, suédois, tchèque et même en ivrite (juive). En Russsie aussi,ces poésies éveillent un grand intérêt. La traduction russe des "chants" et son édition appartient à l'ami de N.G.Tchernichevski, poète M.L. Mikhailov. Après lui, les poésies de Mirzé Chéfi ont été traduites par V.M.Markov, M.Ramch, N.Eyfert, et les autres. Ayant pris les connaissances de ces poésies L.M.Tolstoy les avait bien appréciées. Les poésies de Mirzé Chéfi ont été publiées six fois en 1868, douze fois en 1876. En somme elles ont été publiées 169 fois jusqu'à 1922 . Aucun auteur ne connait un tel succès de publication. Ayant remarqué ce grand succès, en 1873, Bodenstedt trahit à son maître en tâchant de se réclamer le vrai auteur. Orientaliste Adolf Berger en s'appuyant sur les paroles du cheikh-ul-islam de Caucase, mollah Ahmad Salyani, nie absolument le talent de poète et le droit d'auteur de Mirzé Chéfi. en trompant la société littéraire Bodenstedt a dit qu'il avait d'abord édité ses oeuvres sous le nom Mirzé Chéfi, sous l'impression de son voyage à l'Est.

Comme s`il a pris le nom de M. Chafi comme un pseudonyme oriental. Dans les années suivantes Bodenshtedt a pu reunir des admirateurs autour de soi ,alors que pendant ce temps Vazeh a été oublié par le public.Quoiqu il joue un rôle important dans la diffusion des poemes de Vazeh en Europe, il était aussi marqué comme un imitateur de sorte que ca lui a apporté le déshonneur. Il a fait une grande popularité en publiant les poèmes de Vazeh d'une manière privée ,mais il a aussi gagné l’incroyance du public en faisant oublier le nom de Vazeh.

Il est a noter que Bodenshteidtedt a ajouté a ce recueil non seulment les poèmes de Vazeh il a ajouté aussi ceux qui ne lui appartenaient pas.

\section{Déduction}

Malgré la disparition des poèmes de Vazeh en Allemagne, certaines poèmes ont étè trouvé par les chercheurs.Les poèmes trouvés consistent d’une qazelle, un pentametre, un qita et trois couplet en azéri, trois qazelle et deux vers en perse. Parfois on comptait l'auteur de ce poème comme Molla Veli Vidadi en s`appuyant aux fausses sources . Ces poèmes avaient été découvertes pour la première fois par Sultan Mumtaz. Pour étudier les oeuvres de Vazeh profondement et obtenir des résultats concrets, il faut les rechercher en azéri et en perse des sources originaux non pas à travers des traductions. Dans ce recueil il ya le poème de Zuleykha, Mirza Yusifles chansons d'amour, de consolation, de la rose etc. Quoique Vazeh accepte le poète Perse Hafiz comme son maitre, dans ses oeuvres on peut sentir l' esprit, l' âme et la génie des oeuvres de Fuzuli. Pour cela, il faut apprécier Vazeh comme successeur de Fuzuli dans le monde littéraire.

Dans ces modèles on critiquait les religieux qui sont loin de la verité. Pour ça les réligieux ne l'aimaient pas. Les réligieux ne l'aimait guerre car dans ses poèsies M. Chafi utilisait toujours les mots pinards, bien aimées, amante, maitresse etc. Les réligieux ne comprenaient pas que ces mots étaient 
des exemplaires littérraires. Dans les mêmes mots M. Chafi chantait l'amour extraordinaire plus forte que l'amour mondaine. Le premier de ces livres a été redigé pour les élèves supérieurs du lycée et le deuxième était pour les débutant et pour des écoles de province. Deuxième volume a été paru par Ivan Grigoriev a Tabriz en 1855 après la mort de Mirzé Chafi Vazeh et celui-ci fut utilisé, comme un manuel pour apprendre l'azerbaidjanais au lycée et dans les écoles de province.

L'édition complète de ce livre apparut à la fin de l'année 1856 et a envoyé au Ministère de l'Education pour la permission de son utilisation comme un manuel. Mais certains proffesseurs de l'Université de Peterbourg ont écrit des opinions négatives. Dans la deuxième partie on présente des aforizmes et des citations citées de différents auteurs. Elles étaient traduites comme oeuvres Garabakhnamé et Derbendnamé Dans la troisième partie on présente beaucoup de vers. Les guéssidés et les extraits du poème Leyli et Medjnoun y sont introduits aussi. Ainsi, on peut dire que Mirzé Chafi Vazeh a présenté le premier manuel en azerbaidjanais. Après ce manuel à la deuxième cinquantaine du XIX siècle Mirzé Abdulhassan Vézirov, Seyyid Azim Chirvani, Rachid bek Efendiyev ont continué cette traditiond'écrire les manuels pour les écoles. On a fait beaucoup de recherches sur la vie et sur l'activité de Mirzé Chafi Vazeh dans son pays natal et dans des autres pays. En XIX siècle l'orientaliste le Almande Adolf Bergé a fait imprimer un guézel écrit en persanne un tekbeyt (c'est une espèce d'un vers de la poèsie oriantale) de Mirze Chafi Vazeh et la traduction de A. F. Veltmande dans la revue de l'Association Orientalisme d'Allemande.

On a fait la recherche sur M.Ch. Vazeh en Russie, en Europe et dans d'autres pays. On avait imprimé ses oeuvres en les traduisant. Les gens cultivés qui s'intéressaient à la festin "Divani
Hikmet" qu'il avait organisé, ont esayé reunir ses manuscrits. L'un d'eux était Ivan Konstantinoviç Yenipopov. Il a réuni beaucoup de matérieux de $\mathrm{M}$. Vazeh. Hélas on n'avait pas trouvé ces matériaux. Les oeuvres de M.Ch. Vazeh avaient été traduits en géorgien dans les années 60 du XIX siècle. Certains de ces traductions avaientt été imprimés dans le journal Iveriya sous la rédaction de Ilya Tchavtchavadzé. 114 poèsies de M.Ch. Vazeh ont été traduites en géorgien par Akani Gelovani. Proffesseur Aftandil Savadzé a traduit ses deux poésies.

Encore a Tbilissi l'imprimérie "Art" a édité le livre de Akaki Gelovanini qui s'appelle "La symphonie" de safes consacré à M.Ch. Vazeh. Y.Moundelhenk a publié en 1978 à Ha, bourgue le livre "Fridrikh Bodainshtedt et M. Chafidans la critique littéraire azerbaïdjanaise". Après cela beaucoup d'articles sur M. Chafi ont été publié dans la presse allemande. Dans certains oeuvres M. Shafi est reconnu comme grand poète et penseur azerbaïdjanais. En 1967 à Kologne, Yssa Kéhabi originaire de Perse a soutenu une thèse sur le sujet "L'allemagnisation des chants de Hafiz par Fridrikh Bodainshtedt'. D'après l'auteur Mirza Chafi n'était pas poète. Son métier n'était que calligraphe. Mais il oublie qu'au XIXe siècle les azerbaïdjanais étaient reconnus comme turc et tatar. Pour cacher cette vérité il prétend qu'à Tbilisi, (la ville Caucasienne), était impossible l'apprentissage de la langue tatare. Malgré que la tatar était l'une des langues turques il n'était pas la langue parlée au Caucase. C'est pourquoi on n'a pas besoin de l'apprendre à l'école secondaire dans les autres endroits de la Russie. A cause de son antipathie envers les Azerbaidjanais, qu'il crée exprès de fausse imagination dans l'esprit occidentale consernant les noms Azerbaidjanais, Tatars et Turcs.

\section{References:}

1. Aus dem Nachlasse Mirza Schaffy (1875) Berlin, 1875.

2. Bayramov A (2008) Fridrix Bodenştedtin Mirzə Şəfi Vazeh haqqında xatirələri. Bakı: Nurlan, $108 \mathrm{~s}$.

3. Die Lieder des Mirza Schaffy (1851) Berlin, 1851.

4. Zeitschrift für Ethnorogie-Verhandlunge der Berliner Gesellschaft für Anthropologie, (1901) Ethnologie und Urgeschichte. Berlin, 1901.
5. Oliyeva NY (2013) Mirzə Şəfi Vazeh tadqiqatlarında Şərq va Qərb təfəkkürünün vəhdəti. Gəncə: Elm, 231 s.

6. Guliyeva NM, Hasanov EL (2012) New ethnographic approach to the research of main decorative - applied arts of Ganja of the XIX $\mathrm{XX}$ centuries. International scientific conference - Achievements in science: new views, problems, innovations. Lodz. p. 56-58.

7. Guliyeva NM, Hasanov EL (2012) About ethnographic-archaeological research of some 
handicraft branches of Ganja during XIX - XX centuries. Progressive scientific explorations 2012: Proceedings of the $8^{\text {th }}$ International scientific-practical conference. Prague, p. 7375.

8. Häsänov EL (2012) Die Gändschänischen teppiche von XIX - XX Jahrhundert als geschichtliche - ethnographische quelle. European Science and Technology (Die Europäische Wissenschaft und die Technologien): $2^{\text {nd }}$ International scientific conference. Bildungszentrum Rdk e. V. Wiesbaden, p. 26-27.
Impact Factor ESJI (KZ) $\quad=\mathbf{1 . 0 4 2}$

9. Guliyeva NM \& Häsänov EL (2014) Die traditionelle Gändschänischen Teppiche von Zeitraum der Aserbaidschanischen Gelehrten und Dichter Mirsä Schäfi Waseh als ethnoanthropologische Quelle (XIX Jahrhundert). Europäische Fachhochschule, 2, p. 3-5.

10. Nicat Ә (1980) Nă̆məyə dönmüş ömür. Bak1, 1980.

11. Aslanov A (2004) M.Ş.Vazeh. Nağmalar. Bak1: Şərq-Qərb.

12. The dawn of Art (1974) Leningrad: Aurora Art Publishers. $196 \mathrm{p}$.

Elnur Latif oglu Hasanov

membre correspondant del'Académie internationale des sciences théoriques et appliquées,

$\mathrm{Ph}$.D. étudiant de troisième cycle spécialiste de la Direction générale de Gandja de l'Académie Nationale des Sciences d'Azerbaïdjan, Gandja, Azerbaïdjan 1-hasan@hotmail.com 